\title{
STAR heavy-ion highlights
}

\author{
Olga Rusnakova (for the STAR Collaboration) ${ }^{1, a}$ \\ ${ }^{1}$ Czech Technical University in Prague, Faculty of Nuclear Sciences and Physical Engineering, Brehova 7, \\ Praha 1
}

\begin{abstract}
Parton energy loss, quarkonium sequential melting and particle production from electromagnetic interactions are tools to study Quark Gluon Plasma properties. The STAR detector, with large acceptance at mid-rapidity, excellent particle identification and wide transverse momentum coverage, is able to study these probes in details. Di-electron spectra form Beam Energy Scan, measurements of reconstructed jets in Au+Au collisions and quarkonium measurements in $\mathrm{p}+\mathrm{p}$ and heavy ion collisions are reported in this paper.
\end{abstract}

\section{Introduction}

Partons with high transvere momenta $\left(p_{\mathrm{T}}\right)$, which are produced in the hard scatterings of relativistic heavy-ion collisions, interact with the surrounding hot and dense medium, Quark Gluon Plasma (QGP), via color exchange and may lose significant part of their energy. However, single partons can not be measured experimentally. As a proxy, high- $p_{\mathrm{T}}$ hadrons can be used to study parton energy loss. By comparing high- $p_{\mathrm{T}}$ hadrons in heavy-ion and $\mathrm{p}+\mathrm{p}$ collisions, information about parton interactions with the QGP is obtained. The observation of single high- $p_{\mathrm{T}}$ hadron suppression in Au+Au collisions agrees with the parton energy loss expectations [1]. The disappearance of away-side jet peaks in dihadron correlations in $\mathrm{Au}+\mathrm{Au}$ collisions, but not in $\mathrm{d}+\mathrm{Au}$ collisions, further suggests such high$p_{\mathrm{T}}$ hadron suppression is a hot medium effect [2]. However, the leading hadron does not carry all the information of its parent parton. Fully reconstructed jets - collimated sprays of hadrons fragmented and hadronized from a hard scattered parton allow to reconstruct the full kinematics of their parent parton. Therefore they represent a vital probe of the QGP medium. Latest STAR results on reconstructed semi-inclusive jets in Au+Au collisions will be reported in this paper. Another probe used to study the QGP properties besides jets is the quarkonium production. Quarkonia, bound states of quark and anti-quark pairs, produced in the initial hard scattering can be modified by color screening in the QGP and therefore are sensitive to the QGP temperature. In order to study possible quarkonium modification in the QGP, a reference of quarkonium production in $\mathrm{p}+\mathrm{p}$ collisions is needed. Moreover, data on quarkonium production in $\mathrm{p}+\mathrm{p}$ collisions constrain $\mathrm{pQCD}$ calculations $[3,4]$. $\Upsilon$ (bb) suppression in $\mathrm{Au}+\mathrm{Au}$ and $\mathrm{U}+\mathrm{U}$ collisions will be discussed. $\mathrm{J} / \psi(c \bar{c})$ production dependence on event multiplicity at various $p_{\mathrm{T}}$ at mid-rapidity in $\mathrm{p}+\mathrm{p}$ collisions will be also reported. By varying the colliding energy in a wide range (7.7 - $200 \mathrm{GeV}$ per nucleon-nucleon pair), RHIC has conducted a Beam Energy Scan (BES) program to explore the nuclear matter phase diagram. Results from BES - Phase I have shrunk

ae-mail: olga.hajkova@fjfi.cvut.cz 
the region of interest for finding the critical point and the boundary of the first-order phase transition to beam energies below $20 \mathrm{GeV}$.

The STAR experiment comprises several subsystems that provide full azimuthal coverage at midrapidity $(|\eta|<1)$. A detailed description of the STAR detector can be found in [5].

\section{Beam Energy Scan}

In order to explore the QCD phase diagram, a Beam Energy Scan (BES) program was carried out by the STAR experiment at RHIC. A detailed description of the STAR detector can be found in [5]. The main aims of the BES program were to search for the turn-off of QGP signatures, signals of the first order phase transition and the critical point. One of the important QGP signatures is the nuclear modification factor $R_{C P}$ - ratio of yields at most central over peripheral collisions, appropriately scaled by the corresponding number of binary collisions. For several particle species, $R_{C P}$ values less than unity has been measured in heavy ion collisions at high energies [6].

Fig. 1 shows the $R_{C P}$ of charged hadrons in Au+Au collisions at $\sqrt{s_{\mathrm{NN}}}=7.7-200 \mathrm{GeV}$. We observe that for $p_{\mathrm{T}}>2 \mathrm{GeV} / c$, the $R_{C P}$ is less than unity at $39 \mathrm{GeV}$ and then the value increases with the decreasing beam energy.

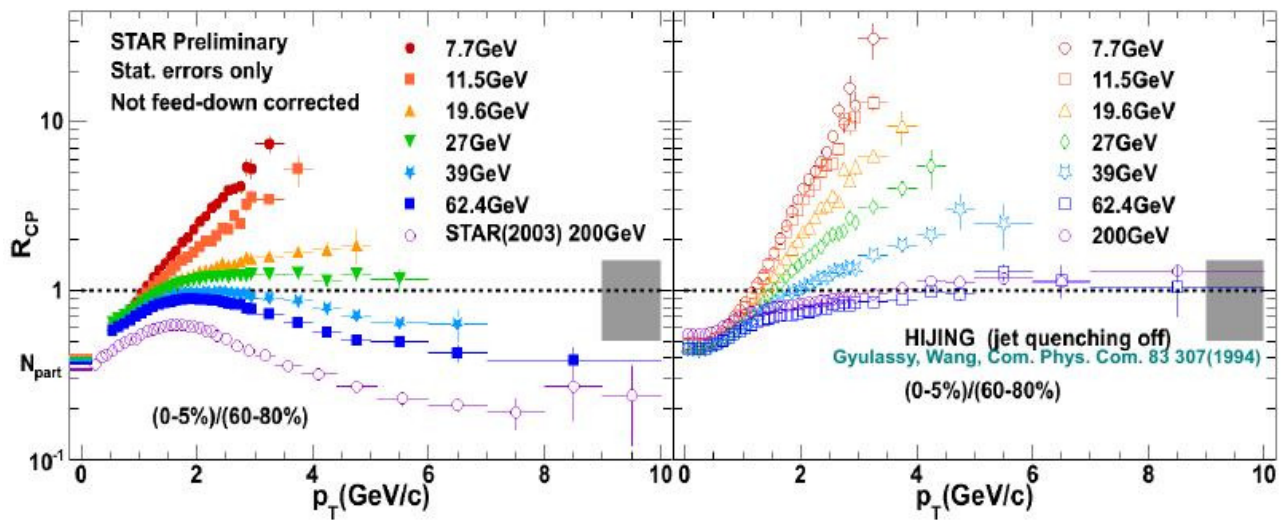

Figure 1. Left: $R_{C P}$ for charged hadrons in $\mathrm{Au}+\mathrm{Au}$ collisions at $\sqrt{s_{\mathrm{NN}}}=7.7-39 \mathrm{GeV}$. Errors are statistical only. Grey band represents the normalization error from $N_{b i n}$. Right: HIJING [7] theoretical prediction of $R_{C P}$ for charged hadrons in $\mathrm{Au}+\mathrm{Au}$ collisions at $\sqrt{s_{\mathrm{NN}}}=7.7-39 \mathrm{GeV}$.

Measurements of QGP radiation would be viable to answer the question for signals of chiral symmetry restoration expected from QCD. Since the in-medium modifications of the spectral functions of short-lived resonances (such as the $\rho$-meson) survive the fireball evolution through the mesons' decays into $e^{+} e^{-}$pairs (di-electrons), low mass di-electron production $\left(<1 \mathrm{GeV} / c^{2}\right)$ is a possible link to chiral symmetry restoration.

As can be seen on Fig. 2, excess yields in the di-electron invariant mass $\left(M_{e^{+} e^{-}}\right)$distributions at $0.2<M_{e^{+} e^{-}}<0.8 \mathrm{GeV} / c^{2}$ over the hadronic decay electron cocktails are observed for collision energies from 19.6 to $200 \mathrm{GeV}$. A model calculation including $\rho$ meson in-medium broadening and chiral symmetry restoration could possibly describe the di-electron spectrum down the enrgy of 20 $\mathrm{GeV}$. Beam energies lower than $20 \mathrm{GeV}$ however require more data for QGP phase transition search in BES - Phase II . 


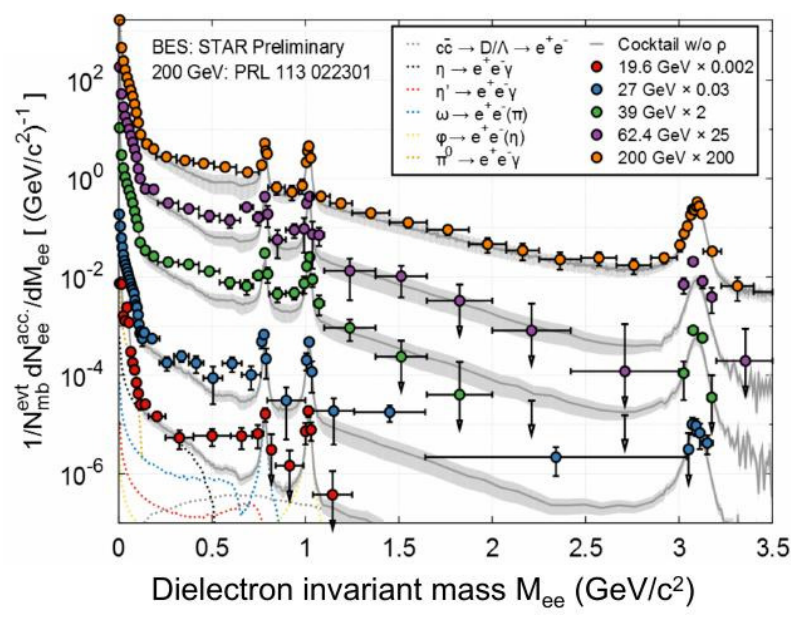

Figure 2. Measured di-electron invariant mass, $M_{e e}(\mathrm{GeV} / \mathrm{c})$, for BES Phase-I compared to cocktail simulations (grey) and model calculations [8]. The single vector meson contributions are depicted for $19 \mathrm{GeV}$. For $39 \mathrm{GeV}$, the two model contributions from hadronic gas and QGP phase are shown next to the total expected yield from cocktail and model (orange). Contributions from photon conversions are not removed from the data at $19 \mathrm{GeV}$.

The first harmonic coefficient of the Fourier expansion of the final-state momentum-space azimuthal distribution relative to the reaction plane is called directed flow $v_{1}$. Both hydrodynamic and nuclear transport models indicate that $v_{1}$ in the mid-rapidity region offers sensitivity to details of the expansion of the participant matter during the early collision stages.

Fig. 3 reports values of $v_{1}(y)$ slope (which is the linear term $F$ in a cubic fit, where $v_{1}(y)=$ $\left.F y+F_{3} y^{3}\right)$ near mid-rapidity for anti-protons, protons and net protons and a comparison with UrQMD transport model. As can be seen, the UrQMD predictions do not describe the data at all.

The observed minimum for protons and net protons is a possible signature of a first-order phase transition between hadronic matter and a deconfined phase.

\section{Semi-Inclusive Charged Jet Measurement}

Jets represent a very valuable probe of the QGP medium because they preserve most of the momenta of the original hard scattered partons. However, jet reconstruction in the environment of heavy-ion collisions has to deal with an immense and highly fluctuating background, resulting in a large number of fake combinatorial jets.

In recoil-jet analysis, the background is not suppressed by a momentum cut on jet constituents. Instead, a hard event is selected by triggering on a high- $p_{\mathrm{T}}$ trigger hadron. An away-side jet is then reconstructed opposite in azimuth

$$
\left|\phi_{\text {Jet }}-\left(\phi_{\text {Trig }}+\pi\right)\right| \leq \pi / 4
$$

Such a jet is completely unbiased with respect to its fragmentation. The semi-inclusive recoil jet yield per trigger is an observable calculable by means of pQCD. In similar recoil jet analyses at the LHC, the background is usually reduced by subtracting corresponding recoil spectra for two different trigger hadron momentum ranges. However, in this analysis an innovative technique of mixed event is 


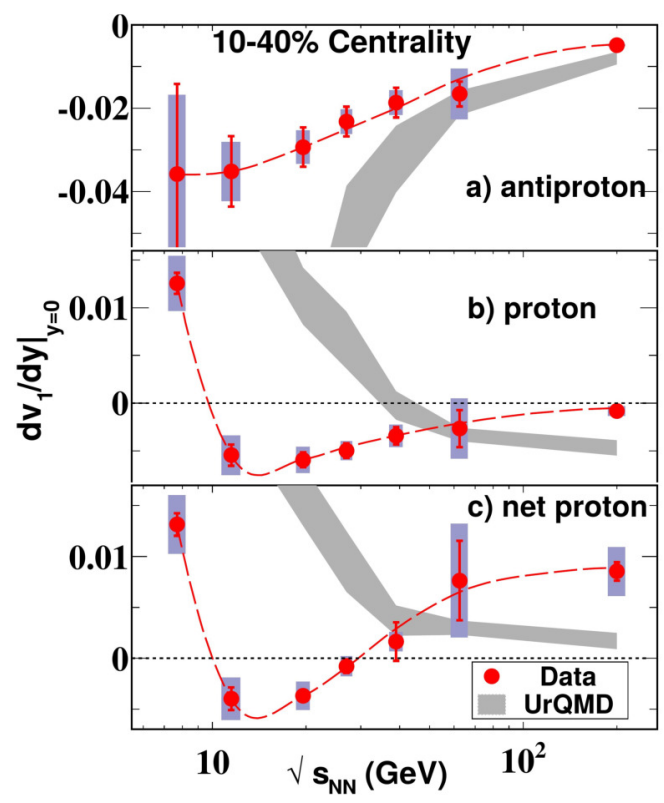

Figure 3. Directed flow slope $\left(\mathrm{d} v_{1} / \mathrm{d} y\right)$ near mid-rapidity versus beam energy for intermediate-centrality $\mathrm{Au}+\mathrm{Au}$. Panels (a), (b) and (c) report measurement for antiprotons, protons, and net protons, respectively, along with UrQMD calculations subject to the same cuts and fit conditions. Systematic uncertainties are shown as shaded bars. Dashed curves are a smooth fit to guide the eye [9].

used to describe the combinatorial background. The mixed event is generated by randomly selecting tracks from real events in the same centrality class, event plane direction and primary vertex $z$ position. All the tracks in the mixed event are therefore fully uncorrelated. By running the jet reconstruction algorithm on the mixed event samples with the same conditions as for real events (with the trigger hadron now chosen in a random direction) a recoil-jet distribution which describes the combinatorial background is obtained.

Analyzed data were recorded with a Minimum Bias (MB) trigger condition in Au+Au collisions at $\sqrt{s_{N N}}=200 \mathrm{GeV}$. Jets were reconstructed from charged tracks measured by the STAR Time Projection CHamber (TPC) using the anti- $\mathrm{k}_{\mathrm{T}}$ sequential clustering algorithm via the FASTJet software package. Jet $p_{\mathrm{T}}$ is then adjusted for background energy on an event-by-event basis according to

$$
p_{\mathrm{T}, \mathrm{jet}}^{\mathrm{reco}}=p_{\mathrm{T}, \mathrm{jet}}-\rho \times A
$$

where $A$ is the jet area calculated using soft ghost particles and $\rho$ is the background energy density calculated for each event as the median jet energy density $\frac{p_{\mathrm{T} \text { jet }}^{i}}{A_{i}}$ from all reconstructed jets in the event, excluding 2 (peripheral) or 3 (central events) hardest jets in the event. The background jets are reconstructed using the $\mathrm{k}_{\mathrm{T}}$ algorithm.

The reconstructed spectra were further corrected for the background fluctuations and detector effects (tracking efficiency, momentum resolution) by the unfolding. Two methods (Bayesian unfolding, 


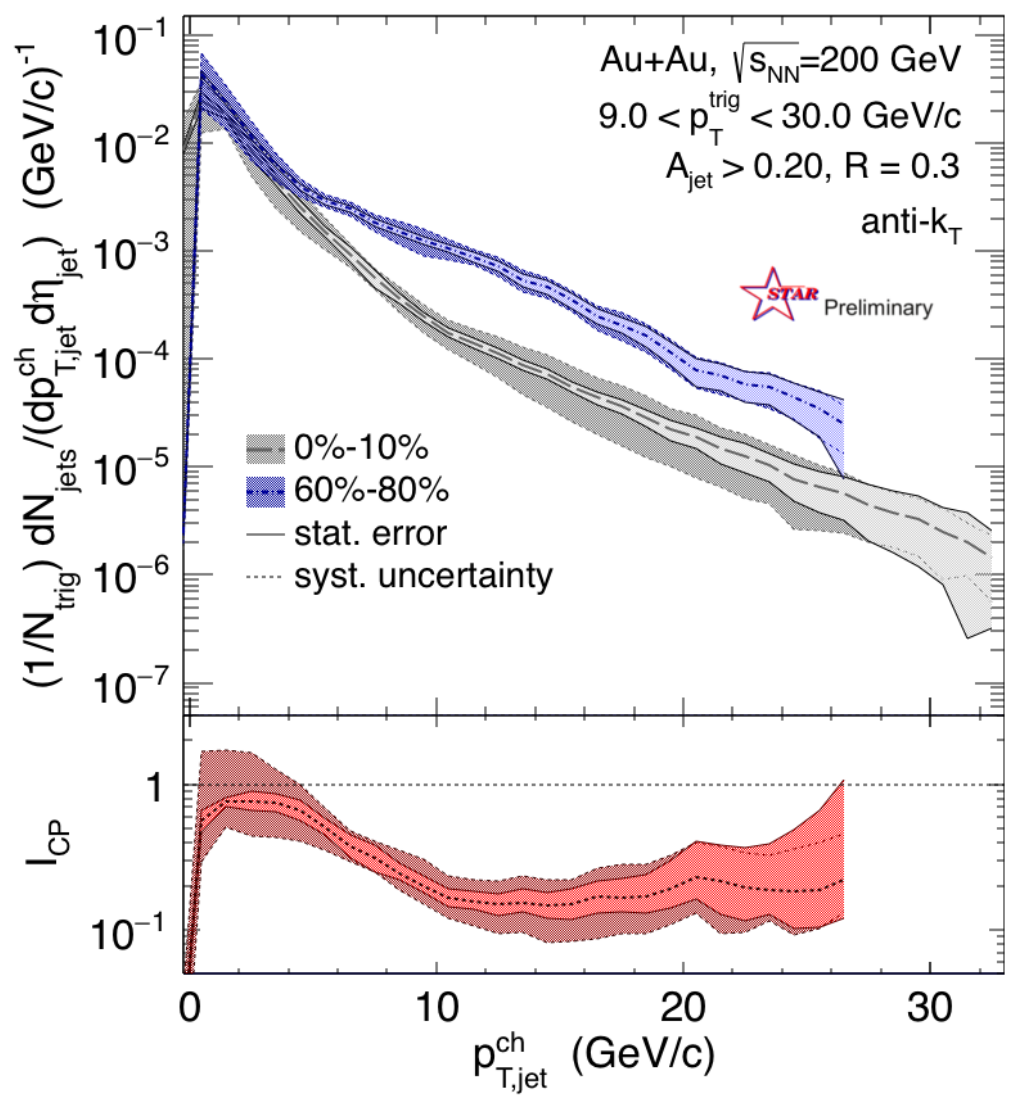

Figure 4. Upper panel: Fully corrected recoil-jet yield per trigger for central and peripheral $\mathrm{Au}+\mathrm{Au}$ collisions at $\sqrt{s_{\mathrm{NN}}}=200 \mathrm{GeV}$. Bottom panel: $I_{C P}$, ratio of fully corrected recoil jet spectra in central (0-10\%) and peripheral $(60-80 \%) \mathrm{Au}+\mathrm{Au}$ collisions at $\sqrt{s_{\mathrm{NN}}}=200 \mathrm{GeV}$.

Singular Value Decomposition [10,11]), several prior distributions and several values of the regularization parameter were used to check the systematic dependency of the unfolding procedure.

In-medium jet modification is measured via the ratio of the recoil yield in central and peripheral collisions $\left(I_{C P}\right)$. Fig. 4 shows the fully corrected spectra both in peripheral and central collisions (top panel) and their ratio $I_{C P}$ (bottom panel). For low jet momenta $p_{\mathrm{T}}<5 \mathrm{GeV} / \mathrm{c}, I_{C P}$ is close to unity. For jet momenta $p_{\mathrm{T}}>10 \mathrm{GeV} / \mathrm{c} I_{C P}$ drops to $\sim 0.2$, which indicates significant jet suppression in central heavy ion collisions compared with peripheral collisions. This is a stronger suppression than observed in a similar measurement by the ALICE collaboration in $\mathrm{Pb}+\mathrm{Pb}$ collisions at $\sqrt{s_{\mathrm{NN}}}=2.76$ TeV. ALICE measured $I_{C P} \approx 0.6$ for $20<p_{\text {, jet }}<100 \mathrm{GeV} / \mathrm{c}$ for charged jets with $R=0.4$ [12]. However the jet $p_{\mathrm{T}}$ shift needed for the peripheral spectra to match the central spectra is similar in 
both experiments, $\approx 8 \mathrm{GeV} / c$. Therefore, the larger suppression observed at RHIC may be due to similar out-of-cone energy transport combined with a steeper falling spectrum at RHIC energies than that at LHC energies. The different choice of trigger particle $p_{\mathrm{T}}$ and collision energies at STAR and ALICE could have an influence on the measured suppression as well.

\section{$4 \Upsilon$ Measurement in $\mathrm{Au}+\mathrm{Au}$ and $\mathrm{U}+\mathrm{U}$ Collisions}

The production of heavy quarkonia, a bound state of a heavy $(c, b)$ quark and its antiquark, is suppressed in heavy ion collisions compared to $\mathrm{p}+\mathrm{p}$ collisions due to the Debye screening of the quark potential. Charmonium $(c \bar{c})$ suppression was therefore proposed as one of the key signatures of the QGP formation [13]. Moreover, it is expected that quarkonium states with different binding energies dissociate at different temperatures, therefore their suppression pattern could serve as a QGP thermometer [14]. However in spite of the original expectations, only a weak energy dependence of $\mathrm{J} / \psi$ suppression has been observed over a broad range of collision energies [15, 16]. An explanation to this is that later recombination of $c \bar{c}$ pairs gives a significant contribution to the $\mathrm{J} / \psi$ yield which compensates for dissociation. Moreover, $\mathrm{d}+\mathrm{Au}$ measurements proved that cold nuclear matter (CNM) effects also play an important role [17]. Feed-down from heavier states such as $\chi_{c}, \Psi_{0}$ and B mesons to $\mathrm{J} / \psi$ also contribute to the measured yields. Bottomonia are expected to be less affected by many of these effects than charmonia, since uncorrelated recombination and co-mover absorbtion are predicted to be negligible at RHIC energies [18]. Therefore bottomonium represents a cleaner probe of the QGP matter. However, a feed-down contribution from sources as $\chi_{b}, \Upsilon(2 S)$ and $\Upsilon(3 S)$ decaying to $\Upsilon(1 S)$ is still present.

Bottomonia $\Upsilon$ were reconstructed in the di-electron decay channel. Fig. 5 shows the $\Upsilon$ nuclear modification factor, $R_{A A}$, at mid-rapidity as a function of number of participants, $N_{\text {part }}$, calculated from the Monte Carlo Glauber Model [19] together with $R_{A A}$ measurements in $\mathrm{Au}+\mathrm{Au}$ collisions at $\sqrt{s_{\mathrm{NN}}}=200 \mathrm{GeV}$ and $R_{A A}$ in U+U collisions at $\sqrt{s_{\mathrm{NN}}}=197 \mathrm{GeV} . R_{A A}$ is close to unity for the collisions with a small number of participants, and significantly suppressed at larger $N_{\text {part }}$. $\mathrm{U}+\mathrm{U}$ collisions produce higher initial energy densities than Au+Au collisions since the U-nucleus has a larger number of nucleons than an Au-nucleus. The measured $U+U$ data confirm the $\Upsilon$ suppression seen in $\mathrm{Au}+\mathrm{Au}$ collisions and extend the $R_{A A}$ measurement to higher $N_{\text {part }}$. To test the sequential melting of the different $\Upsilon$ states, more statistics would be needed. Nevertheless, the emerging pattern of increasing suppression with higher $N_{\text {part }}$ and lower binding energy is consistent with the sequential melting hypothesis.

The $\mathrm{Au}+\mathrm{Au} \Upsilon(1 \mathrm{~S})$ shows a suppression similar to that of high- $p_{T} \mathrm{~J} / \psi$ mesons, which can be only explained if also cold nuclear matter effects were present [20]. In centrality integrated $\mathrm{Au}+\mathrm{Au}$ collisions the $(2 \mathrm{~S}+3 \mathrm{~S})$ state yields are consistent with a complete suppression within the measurement error. Although the $\mathrm{U}+\mathrm{U}$ results are consistent with $\mathrm{Au}+\mathrm{Au}$, the significance that $R_{A A}>0$ for the $\Upsilon(2 \mathrm{~S}+3 \mathrm{~S})$ states in $\mathrm{U}+\mathrm{U}$ collisions of $0-60 \%$ centrality is $1.8 \sigma$.

Even though comparable with each other, results form heavy ion collisions at RHIC exhibit a steeper $N_{\text {part }}$ dependence than results from $\mathrm{Pb}+\mathrm{Pb}$ collisions at $2.76 \mathrm{TeV}$ at the LHC [21]. This may suggest that suppression is driven by the energy density and not strictly by the number of participant nucleons.

The model of Strickland and Bazow [22] incorporates lattice QCD results on bottomonium screening and thermal broadening, as well as the dynamical propagation of the $\Upsilon$ meson in the colored medium. Assuming an initial central temperature $428<T<444 \mathrm{MeV}$, the scenario with a potential based on heavy quark internal energy is consistent with the observations, while the free energy based scenario is disfavoured. The model of Liu et al. [23] uses an internal-energy-based potential and 

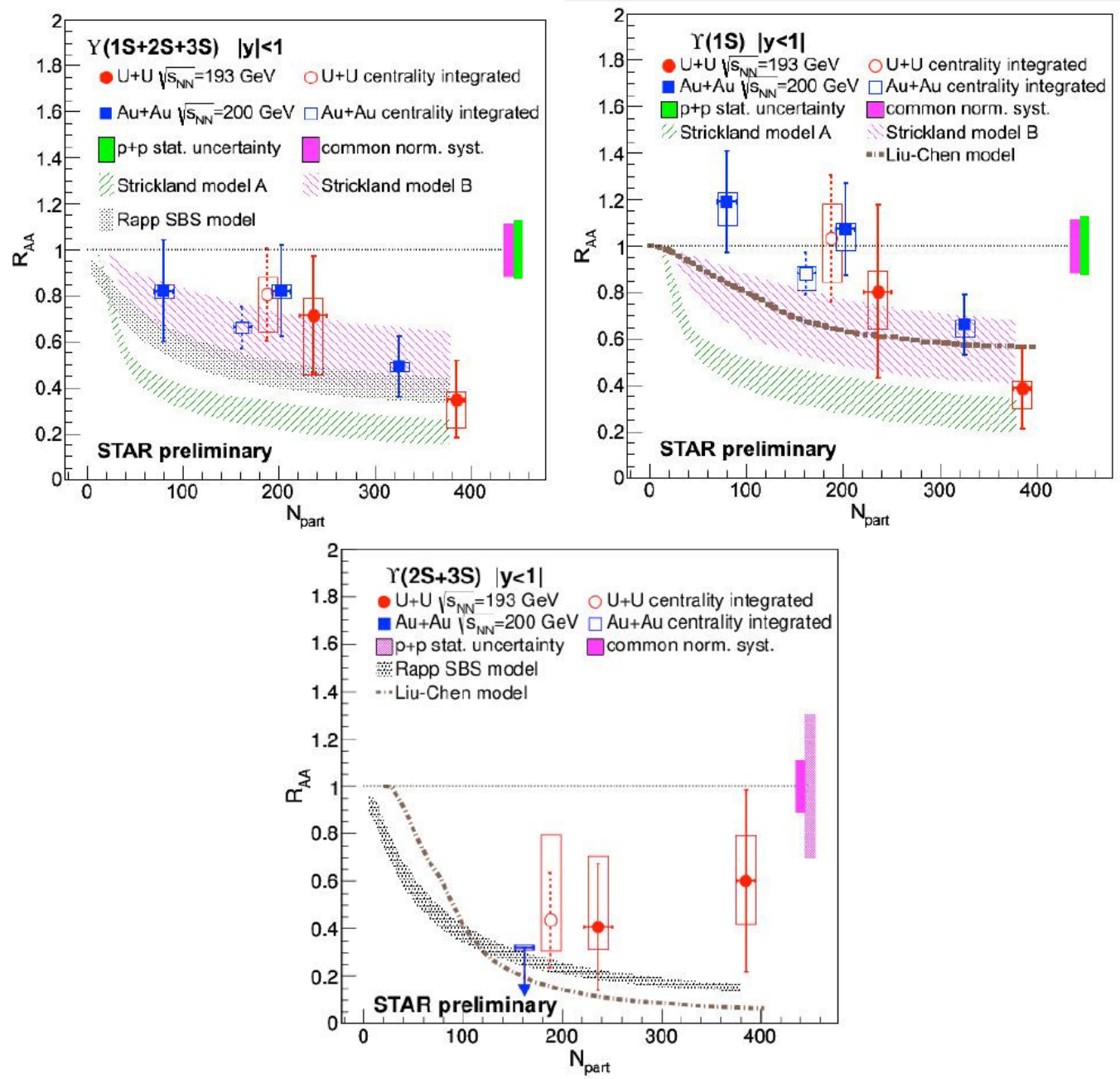

Figure 5. $R_{A A}$ of the $\Upsilon(1 \mathrm{~S}+2 \mathrm{~S}+3 \mathrm{~S})$ (left), $\Upsilon(2 \mathrm{~S}+3 \mathrm{~S})$ (bottom) and the $\Upsilon(1 \mathrm{~S})$ (right) states plotted as a function of $N_{\text {part }}$, for two collision energies and systems: $\sqrt{s_{\mathrm{NN}}}=200 \mathrm{GeV} \mathrm{Au}+\mathrm{Au}$ (squares) and $\sqrt{s_{\mathrm{NN}}}=193 \mathrm{GeV} \mathrm{U}+\mathrm{U}$ collisions (circles), compared to different models. Open symbols represent the measurements of 0-60\% centrality. The models on the bottom figure are for $\Upsilon(2 S)$ only.

an input temperature $\mathrm{T}=340 \mathrm{MeV}$. The model proposed by Emerick, Zhao, and Rapp [24], which includes possible CNM effects in addition, is also consistent with STAR results.

We observe a significant suppression of bottomonium states in central heavy ion collisions. While the $\Upsilon(1 \mathrm{~S})$ is similarly suppressed in $\sqrt{s_{\mathrm{NN}}}=200 \mathrm{GeV} \mathrm{Au}+\mathrm{Au}$ collisions to the high- $p_{T} \mathrm{~J} / \psi$ mesons, there is a stronger suppression in the case of the excited states $\Upsilon(2 S+3 S)$. This attests to the sequential melting picture in the presence of a deconfined medium. The suppression pattern seen in the case of the $\mathrm{U}+\mathrm{U}$ collisions is consistent with the trend marked in $\mathrm{Au}+\mathrm{Au}$ collisons and extends it towards higher $N_{\text {part }}$ values. The indication of $\Upsilon(1 \mathrm{~S})$ suppression in central $\mathrm{Au}+\mathrm{Au}$ collisions is confirmed by the new measurements in $\mathrm{U}+\mathrm{U}$. There is a hint with an $1.8 \sigma$ significance that $\Upsilon(2 \mathrm{~S}+3 \mathrm{~S})$ is not 
completely suppressed in 0-60\% U+U collisions at $193 \mathrm{GeV}$. An ongoing analys of high statistics 2014 data will reduce statistical and systematical errors significantly and should provide separate $R_{A A}$ measurements of all three $\Upsilon(1 \mathrm{~S}), \Upsilon(2 \mathrm{~S})$ and $\Upsilon(3 \mathrm{~S})$ states [25]. High statistics p+Au collisions taken in 2015 will help us gain a deeper insight to the CNM effects.

\section{$5 \mathrm{~J} / \psi$ Measurement in $\mathrm{Au}+\mathrm{Au}$ and $\mathrm{U}+\mathrm{U}$ Collisions}

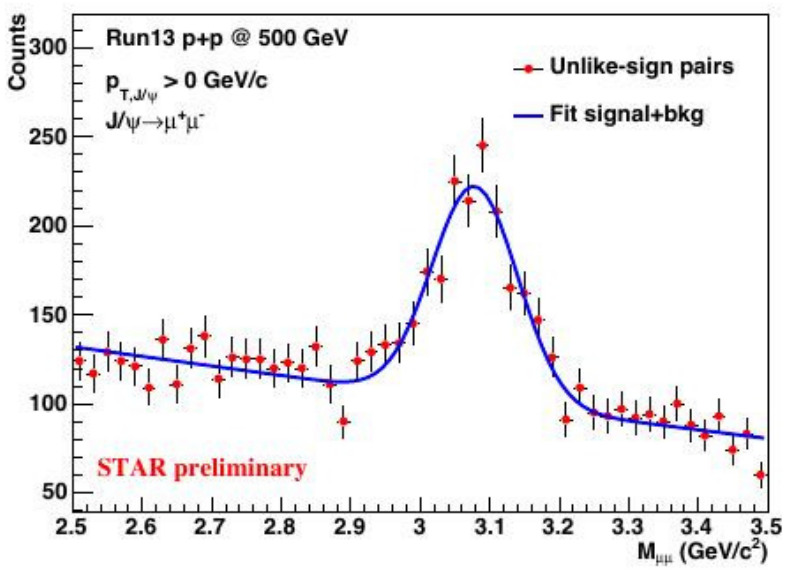

Figure 6. Invariant mass distribution of unlike-sign muon pairs (red circles) fitted with a Gaussian function describing signal and an exponential function describing background (solid line).

Measurements of $\mathrm{J} / \psi$ yields in $\mathrm{p}+\mathrm{p}$ collisions serve as the baseline for heavy-ion collision measurements and can also constrain theoretical models of different production mechanisms [3, 4]. Recent reports from the LHC on $\Upsilon$ yields [26] and on D meson and J/ $\psi$ yields [27] showed enhancement of charm and bottom production in high event activity $\mathrm{p}+\mathrm{p}$ collisions.

STAR measured $\mathrm{J} / \psi$ production from the di-electron and also in the di-muon decay channel. Fig. 6 shows the invariant mass peak of $\mathrm{J} / \psi$ meson reconstructed in the di-muon decay channel using the recently installed MTD detector. Fig. 7 shows relative $\mathrm{J} / \psi$ yield as a function of event activity in $\mathrm{p}+\mathrm{p}$ collisions at $\sqrt{s}=500 \mathrm{GeV}$. The event activity is characterized by the number of charged particles in pseudorapidity range $|\eta|<0.9$ relative to that in a MB $\mathrm{p}+\mathrm{p}$ event. Red points show $\mathrm{J} / \psi$ production from the di-muon channel with $|\eta|<0.5$ and $p_{T}>0 \mathrm{GeV} / \mathrm{c}$ and blue points show $\mathrm{J} / \psi$ from the di-electron channel with $|\eta|<1$ and $p_{T}>4 \mathrm{GeV} / \mathrm{c}$. The black dashed line represents the linear case of a ratio of $\mathrm{J} / \psi$ production to the charged particle multiplicity. The low- $p_{\mathrm{T}}$ di-muon $\mathrm{J} / \psi$ yield roughly follows the dashed line while the high- $p_{\mathrm{T}}$ di-electron $\mathrm{J} / \psi$ yield rises faster than the linear trend. Enhancement of $\mathrm{J} / \psi$ production in the high activity events implies that hard processes dominate over soft processes in high multiplicity $\mathrm{p}+\mathrm{p}$ collisions relative to low multiplicity collisions. The PYTHIA8 predictions for $\mathrm{J} / \psi p_{T}$ above 0 and $4 \mathrm{GeV} / \mathrm{c}$ are shown as the dashed lines. The shaded areas represent the statistical errors. The percolation model [28] is plotted as a solid curve. For $\mathrm{J} / \psi$ with $p_{T}>0 \mathrm{GeV} / \mathrm{c}$ the two models exhibit different trends in the area where the event multiplicity is 2 times higher than the MB multiplicity. The year $2015 \mathrm{p}+\mathrm{p}$ collision data will bring more statistics which will allow to discriminate the models. 

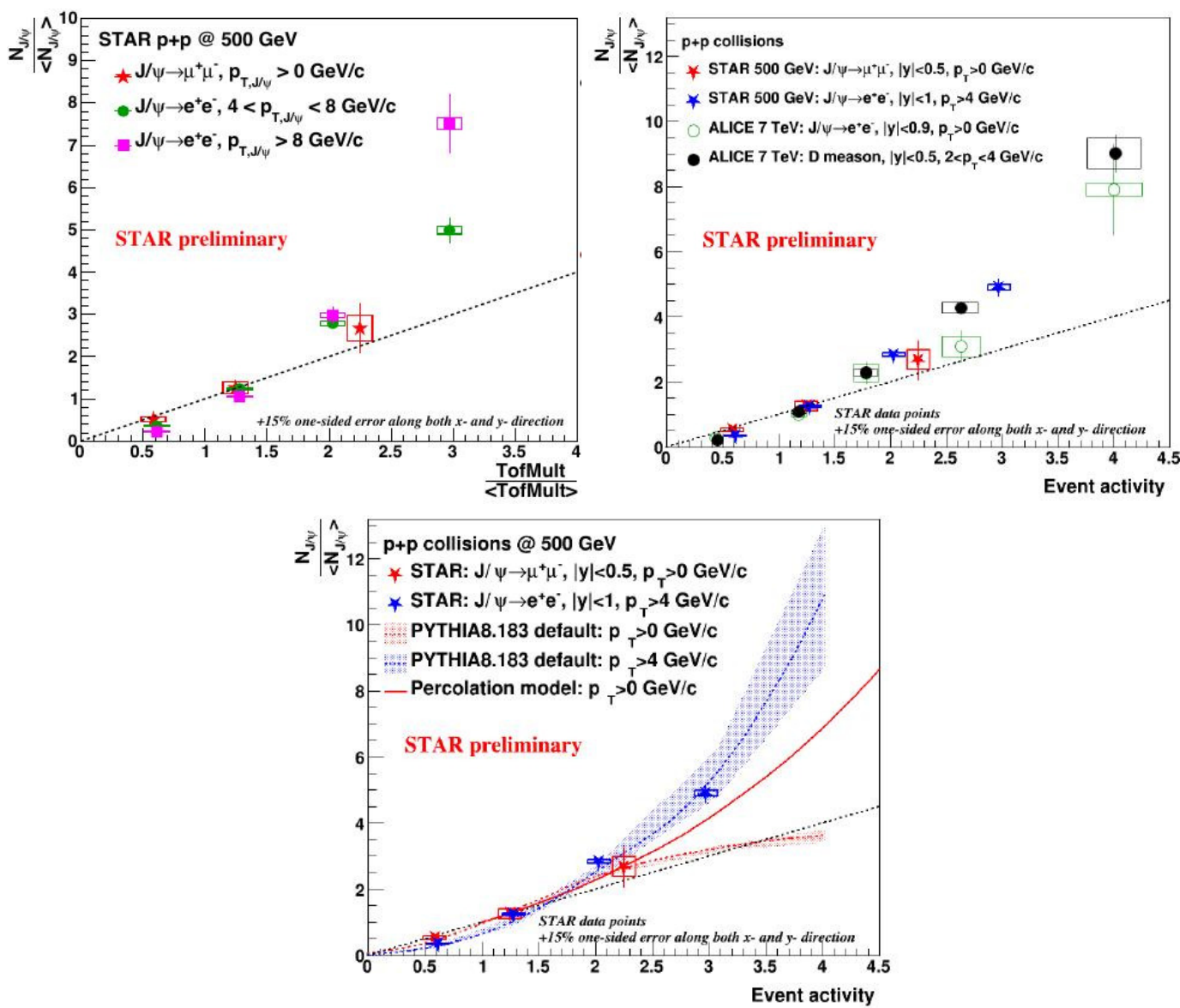

Figure 7. Dependence of relative invariant $\mathrm{J} / \psi$ yield on the event activity: Left: For three different $\mathrm{J} / \psi p_{\mathrm{T}} \mathrm{bins}$. Bottom: For STAR measurement, PYTHIA8 prediction and the percolation model. Right: For STAR (500 GeV) and ALICE $(7 \mathrm{TeV})$ measurements.

\section{Summary and Outlook}

In summary, larger jet suppression has been measured at RHIC energies than LHC energies for semiinclusive charged jets, which however corresponds to a similar horizontal $p_{\mathrm{T}}$ shift in the jet spectrum.

$\Upsilon$ suppression in higher energy density $\mathrm{U}+\mathrm{U}$ collisions confirms and extends the trend seen in $\mathrm{Au}+\mathrm{Au}$ collisions. The precision of current measurements in the di-electron channel is however limited by the low statistics of the heavy ion and the reference samples. Ongoing analysis of the highstatistics $\sqrt{s_{\mathrm{NN}}}=200 \mathrm{GeV} \mathrm{Au}+\mathrm{Au}$ dataset, taken in 2014 with the recently installed MTD detector, will strongly reduce both the statistical and systematic uncertainties by utilizing the di-muon decay channel. The event activity dependence of $\mathrm{J} / \psi$ production in $\mathrm{p}+\mathrm{p}$ collisions is observed.

In 2014, STAR underwent two major heavy flavor detector upgrades, the MTD, to identify muons from quarkonium decay, and the HFT to precisely measure displaced secondary vertices of mesons and baryons containing heavy quarks. In 2015-2017, with STAR's enhanced capabilities in heavy 
flavor measurement, high multiplicity $\mathrm{p}+\mathrm{p}$ and $\mathrm{p}+\mathrm{Au}$ collisions will be investigated, and information on the QGP temperature and shear viscosity in $\mathrm{Au}+\mathrm{Au}$ collisions at $200 \mathrm{GeV}$ are also expected to come. In 2019-2020, RHIC will have a dedicated program for BES - Phase II with sufficiently high luminosity for 5-20 GeV Au+Au collisions. STAR will also carry out an inner-TPC (iTPC) upgrade, which will extend the pseudo-rapidity coverage, increase low $p_{\mathrm{T}}$ reach in tracking of particles for QGP bulk property study, and improve the $\mathrm{dE} / \mathrm{dx}$ resolution of proton/kaon separations at high $p_{\mathrm{T}}$ for jet fragmentation measurements, in addition to the electron identification and acceptance for the low mass di-electron studies discussed above. The refined high-precision measurements will enable BES

- Phase II to boost our understanding of the nuclear matter phase diagram.

\section{References}

[1] J. Adams et al. (STAR), Phys. Rev. Lett. 91, 172302 (2003), nucl-ex/0305015

[2] J. Adams et al. (STAR), Phys. Rev. Lett. 91, 072304 (2003), nucl-ex/0306024

[3] M. Butenschoen, B.A. Kniehl, Phys. Rev. D84, 051501 (2011), 1105.0820

[4] Y.Q. Ma, R. Venugopalan, Phys. Rev. Lett. 113, 192301 (2014)

[5] K.H. Ackermann et al. (STAR), Nucl. Instrum. Meth. A499, 624 (2003)

[6] J. Adams et al. (STAR), Nucl. Phys. A757, 102 (2005), nucl-ex/0501009

[7] M. Gyulassy, X.N. Wang, Comput. Phys. Commun. 83, 307 (1994), nucl-th/9502021

[8] R. Rapp, J. Wambach, Adv. Nucl. Phys. 25, 1 (2000), hep-ph/9909229

[9] L. Adamczyk et al. (STAR), Phys. Rev. Lett. 112, 162301 (2014), 1401.3043

[10] G. D’Agostini, ArXiv e-prints (2010), 1010.0632

[11] A. Hocker, V. Kartvelishvili, Nucl.Instrum.Meth. A372, 469 (1996), hep-ph/9509307

[12] J. Adam et al. (ALICE), JHEP 09, 170 (2015), 1506.03984

[13] T. MATSUI, H. Satz, PHYSICS LETTERS B 178, 416 (1986)

[14] A. Mócsy, P. Petreczky, Phys. Rev. Lett. 99, 211602 (2007)

[15] B. Alessandro et al. (NA50), Eur. Phys. J. C39, 335 (2005), hep-ex/0412036

[16] A. Adare et al. (PHENIX), Phys. Rev. Lett. 98, 232301 (2007), nucl-ex/0611020

[17] A. Adare et al. (PHENIX Collaboration), Phys. Rev. Lett. 107, 142301 (2011)

[18] Z. Lin, C. Ko, Physics Letters B 503, 104 (2001)

[19] R.J. Glauber, Nuclear Physics A 774, 3 (2006), \{QUARK\} \{MATTER\} 2005Proceedings of the 18th International Conference on Ultra-Relativistic Nucleus-Nucleus Collisions

[20] L. Adamczyk et al. (STAR), Phys. Lett. B735, 127 (2014), [Erratum: Phys. Lett.B743,537(2015)], 1312.3675

[21] S. Chatrchyan et al. (CMS Collaboration), Phys. Rev. Lett. 109, 222301 (2012)

[22] M. Strickland, D. Bazow, Nucl. Phys. A879, 25 (2012), 1112. 2761

[23] Y. Liu, B. Chen, N. Xu, P. Zhuang, Physics Letters B 697, 32 (2011)

[24] A. Emerick, X. Zhao, R. Rapp, Eur. Phys. J. A48, 72 (2012), 1111.6537

[25] C. Yang et al., Nucl. Instrum. Meth. A762, 1 (2014), 1402. 1078

[26] S. Chatrchyan et al. (CMS), JHEP 04, 103 (2014), 1312.6300

[27] J. Adam et al. (ALICE), JHEP 09, 148 (2015), 1505 . 00664

[28] E.G. Ferreiro, C. Pajares, Phys. Rev. C 86, 034903 (2012) 